\title{
El expresivismo clásico y los límites de la interpretación naturalista de la intencionalidad en el programa de Grice
}

\author{
Manuel Almagro Holgado \\ Universidad de Granada
}

Resumen: Las consideraciones del filósofo Herbert Paul Grice en el campo de la pragmática marcaron un punto de inflexión en la historia de la filosofia. Ellas siguen siendo de enorme utilidad, y la interpretación de sus escritos está abierta a discusión. Sin embargo, el análisis de Grice en términos de las intenciones de los hablantes ha dado pie a que se interprete su programa como una postura compatible con el naturalismo de la intencionalidad. Sin embargo, esta interpretación tiene serios problemas. En el presente trabajo presentaremos la posición de Grice y la interpretación naturalista de su diagnóstico, señalaremos las objeciones a esta última desde el expresivismo clásico, y defenderemos que puede hacerse una interpretación de Grice más consistente con su pensamiento y capaz de esquivar las críticas del expresivismo.

Palabras clave: Grice; naturalismo; expresivismo clásico; intencionalidad; normatividad

Abstract: "Classical Expressivism and the Limits of the Naturalistic Interpretation of Intentionality in Grice's Program". Grice's ideas in the field of pragmatics were a turning point in the history of philosophy: they are still of enormous utility and the interpretation of his writings is still open to discussion. However, Grice's analysis of meaning based on the intentions of the speakers gave room to a naturalistic interpretation of his account, which gives rise to serious problems. In this article, Grice's account and the naturalistic interpretation of his assessment will be exposed and the objections from classical expressivism will be pointed out. Finally, it will be argued that it is possible to offer a more faithful interpretation, capable of avoiding the criticisms made by the expressivists.

Keywords: Grice; naturalism; classical expressivism; intentionality; normativity 


\section{Introducción}

Una de las contribuciones filosóficas más importantes en el campo de la pragmática en el siglo XX se la debemos al filósofo británico Herbert Paul Grice. El pragmatismo como tal surgió de las consideraciones filosóficas del Wittgenstein maduro, a saber, que el significado no debe explicarse en términos de la relación entre el lenguaje y la realidad, sino apelando al uso. Partiendo del análisis de lo que hacemos con el lenguaje cuando nos comunicamos, podemos distinguir dos vías distintas que se han desarrollado extensamente a lo largo de la historia. Una de ellas, iniciada por Austin, se centra en la dependencia del significado con cierta normatividad. La segunda, que tiene su origen en Grice, introduce una restricción más radical: el significado puede explicarse en términos de las intenciones del hablante.

La preocupación general de Grice fue la naturaleza del significado y sus consideraciones al respecto han abonado el terreno de numerosas propuestas filosóficas. Las importantes obras Relevance de Sperber y Wilson ${ }^{1}$ y Linguistic Communication and Speech Acts de Bach y Harnish ${ }^{2}$ sirven como ejemplos de teorias deudoras de las consideraciones griceanas. En este sentido, Grice abrió una nueva línea de investigación dentro del campo de la pragmática: el enfoque intencionalista.

En cuanto al análisis propuesto por Grice, pueden hacerse, como veremos, al menos dos interpretaciones. De acuerdo con Stalnaker ${ }^{3}$, la reducción griceana de la noción de significado a la noción de intención encaja bien con los objetivos del naturalismo de la intencionalidad. La tesis fundamental de esta interpretación es que el programa de Grice es compatible con un naturalismo reduccionista, articulándose en torno a dos ideas: (i) que los fenómenos intencionales son independientes de cualquier convención, (ii) que dichos fenómenos intencionales pueden ser explicados en términos de la ciencia. Así entendida, la reducción de la semántica a la intencionalidad defiende que las intenciones de los hablantes pueden explicarse en términos científico-descriptivos. Sirven

\footnotetext{
Sperber, D. y D. Wilson, Relevance: Communication and Cognition, Oxford: Blackwell, 1986. Bach, K. y R. Harnish, Linguistic Communication and Speech Acts, Cambridge: MIT Press, 1982. Stalnaker, R., Inquiry, Cambridge: MIT Press, 1984.
} 
como ejemplos de naturalismo de la intencionalidad las propuestas de Fodor ${ }^{4}$ y Stalnaker ${ }^{5}$. Bajo esta interpretación, el diagnóstico que Grice ofrece de la noción de significado ocasional del hablante puede reducirse a una explicación naturalista de las intenciones del hablante.

Sin embargo, esta interpretación se encuentra con problemas serios. Por una parte, filósofos como David Lewis ${ }^{6}$ y Max Kölbel ${ }^{7}$ han defendido de un modo convincente que se requiere una noción social o convencional, más que una noción psicológica, para explicar la comunicación. Por otro lado, el expresivismo clásico mantiene que hay ciertas expresiones del lenguaje que no pueden reducirse a un conjunto de descripciones. Filósofos como Moore ${ }^{8}$, Ayer ${ }^{9}$, Stevenson ${ }^{10}$ y Wittgenstein ${ }^{11}$ hicieron hincapié en que el uso de algunas expresiones de nuestro lenguaje no describe nada del mundo, sino que expresan sentimientos o estados mentales. El programa de Grice queda abatido tras estas criticas, pero ello no significa que las cosas queden alli.

Después de explicar los puntos relevantes de la teoría de Grice para nuestros intereses (sección 2), exponer más detenidamente en qué consiste la interpretación naturalista de su programa (sección 3) y señalar las limitaciones de esta interpretación desde el punto de vista del expresivismo clásico (sección 4), argumentaremos que, rescatando uno de los principios fundamentales en la teoria de Grice -el Principio de Cooperación-, es posible otra interpretación incompatible con la interpretación naturalista de su proyecto. Defenderemos esto último a partir de una nueva formulación de sus consideraciones hecha bajo una luz normativa. Desde nuestra interpretación, las críticas del expresivismo y los problemas con los que se encuentra el naturalismo quedan desactivados. Desarrollaremos más extensamente esta propuesta y señalaremos algunos pasajes del trabajo del propio Grice que servirán como apoyo a nuestra tesis (sección 5).

\footnotetext{
Fodor, J., A Theory of Content and other Essays, Cambridge: MIT Press, 1990.

Stalnaker, R., Inquiry.

Lewis, D., Convention, Cambridge: Harvard University Press, 1969.

Kölbel, M., "Conversational Score, Assertion, and Testimony", en: Brown, J. y H. Cappelen (eds.), Assertion: New Philosophical Essays, Oxford: Oxford University Press, 2011, pp. 49-77.

8 Moore, G.E., Principia Ethica, Nueva York: Prometheus Books, 1988.

9 Ayer, A.J., Lenguaje, Verdad y Lógica, Suárez, M. (trad.), Barcelona: Ediciones Orbis, 1984.

10 Stevenson, C.L., "The Emotive Meaning of Ethical Terms", en: Mind. New Series, v. XLVI, 181 (1937), pp. 14-31.

${ }^{11}$ Wittgenstein, L., Tractatus logico-philosophicus, Muñóz, J. y I. Reguera (trads.), Madrid: Alianza Editorial, 1957; Investigaciones Filosóficas, García Suárez, A. y C.U. Moulines (trads.), México: Universidad Nacional Autónoma de México, 1986; Zettel, Castro, O. y C.U. Moulines (trads.), México: Universidad Nacional Autónoma de México, 1997.
} 


\section{El programa de Grice}

La preocupación general de Grice fue la naturaleza del significado. Concretamente, le interesó "qué es lo que significan los actos, hechos u objetos significativos, sean o no lingüísticos, y cómo logran significar aquello que significan" ${ }^{12}$. Su contribución más significativa fue probablemente su famosa distinción entre lo que un hablante dice y lo que quiere decir con lo que dice. Esta distinción apunta en la dirección de esclarecer dos dimensiones del significado: tenemos, por una parte, el significado de una proferencia $p \mathrm{y}$, por otra, lo que un hablante quiere significar cuando emite $p$. La distinción entre el significado de la proferencia y el significado del hablante supone uno de los puntos de partida fundamentales para la teoría de Grice, pues, en palabras suyas: "el significado de un signo necesita explicarse en términos de lo que quienes lo utilizan quieren decir (o deberian querer decir) con él en ocasiones particulares" ${ }^{13}$. Las nociones fundamentales de su programa son el significado ocasional del hablante y la intención comunicativa. Reconstruiremos aquí únicamente, debido a nuestros intereses, dos elementos relevantes de su diagnóstico para explicar las condiciones que posibilitan que un hablante quiera decir algo mediante una proferencia: la intencionalidad y la racionalidad. Con "intencionalidad" nos referimos al modo en que se analiza el significado del hablante (significado no natural) en términos de intenciones. Con "racionalidad" apuntamos a uno de los presupuestos que hay en toda conversación según Grice: tener una actitud cooperativa.

En su artículo "Significado" de 1957, Grice se preguntó por los distintos sentidos del término "significa" y distinguió entre el sentido natural (SN) y el sentido no natural (SNN). La distinción entre SN y SNN de "significa" no es exhaustiva, como el filósofo reconoce, pero "en la mayoría de los casos deberíamos al menos estar fuertemente inclinados a asimilar un uso de 'significa' a un grupo más que al otro"14. Tres rasgos fundamentales para distinguir entre ambos sentidos son los siguientes: (i) si $x$ significa naturalmente $p$, entonces $x$ implica $p$; si $x$ significa no naturalmente $p$, entonces $x$ no implica $p$; (ii) si $x$ significa naturalmente $p$, entonces " $x$ significa $p$ " no puede reelaborarse de manera que lo que sigue al verbo vaya entrecomillado; si $x$ significa no naturalmente

\footnotetext{
${ }^{12}$ Frápolli, M.J. y E. Romero, Una aproximación a la filosofia del lenguaje, Madrid: Síntesis, 1998, p. 173.

${ }^{13}$ Grice, H.P., “Meaning”, en: The Philosophical Review, v. LXVI, 3 (1957), pp. 377-388; traducción: "Significado", en Valdés, L. (ed.), La búsqueda del significado, Madrid: Tecnos, 2005, p. 484 ${ }^{14}$ Ibid., p. 483.
} 
$p$, entonces puede reelaborarse de manera que lo que sigue al verbo vaya entrecomillado; (iii) si $x$ significa naturalmente $p$, entonces $x$ no quiere decir intencionadamente nada; si $x$ significa no naturalmente $p$, entonces alguien quiso decir intencionadamente algo con $x$.

La cláusula (iii) es algo más problemática debido a que Grice, por conveniencia, incluyó bajo el SN de "significa" aquellos casos que puedan "ejemplificarse en oraciones del siguiente patrón: 'A pretende (pretendia) hacer tal o cual cosa (mediante $x$ )' "15; en estos, él cree que hay intencionalidad. De acuerdo con Grice, serán casos del SNN de "significa" aquellos que puedan presentarse con el patrón " $H$ (un hablante) significa (quiere decir) algo mediante $x$ ".

En su trabajo de 1969, "Las intenciones y el significado del hablante", llevó a cabo un análisis más profundo del SNN de "significa", distinguiendo entre el significado atemporal de una proferencia-tipo (completa o incompleta), el significado atemporal aplicado de una proferencia-tipo (completa o incompleta), el significado ocasional de una proferencia-tipo, y el significado ocasional del hablante ${ }^{16}$. Para aclarar estos elementos, utilizó como ejemplo la oración $O$ : "si mantengo los circuitos abiertos, podría recibir la noticia". Según Grice ${ }^{17}, O$ puede significar tanto la oración "si mantengo abiertas las líneas, podría recibir la noticia", como la oración "si mantengo abiertas las pistas para competiciones de vehículos a motor, podría recibir la noticia". Estas oraciones forman parte del significado atemporal de la proferencia-tipo. Entonces, si vinculamos a $O$ uno de estos significados atemporales de la proferencia-tipo, excluyendo los demás, tendríamos como resultado el significado atemporal aplicado de la proferencia-tipo, esto es, la elección de uno de los significados atemporales posibles. Pero cabe otra posibilidad. Cuando un hablante $H$ profiere $O$ quizás quiere decir, mediante $O$, lo siguiente: "si Dios existe y no dejo de rezarle, me ayudará". En este caso, la oración "si Dios existe y no dejo de rezarle, me ayudará" es el significado ocasional de la proferencia-tipo O. Estos tres tipos hasta ahora mencionados son radicalmente distintos con respecto al cuarto. Con este último ya no se habla del significado de lo proferido, sino de lo que $H$ quiso decir con lo proferido. Así, si cuando $H$ profirió $O$ quiso decir que si Dios existe y no deja de rezarle, le ayudará, entonces este es el significado ocasional

\footnotetext{
${ }^{15}$ Ibid.

${ }^{16}$ Grice, H.P., "Utterer's Meaning and Intentions", en: The Philosophical Review, v. LXXVIII, 2 (1969), pp. 147-177; traducción: "Las Intenciones y el Significado del Hablante", en: Valdés, L. (ed.), La búsqueda del significado, Madrid: Tecnos, 2005, p. 492.

${ }_{17}$ Ibid., pp. 491-494.
} 
del hablante. El significado ocasional de la proferencia-tipo y el significado ocasional del hablante se diferencian en que en el segundo no hablamos del significado de lo que se ha dicho, sino de lo que el hablante quería decir con lo dicho. Aquí encontramos lo que al principio de la sección decíamos que quizás sea la contribución más significativa de Grice, a saber, la distinción entre lo que un hablante dice y lo que quiere decir.

Ahora bien, ¿cuáles son las condiciones que deben darse para que haya significado no natural? Retomando lo que decíamos más arriba: ¿qué tiene que ocurrir para que un hablante diga lo que quiere decir con lo que dice? ¿Cuáles son los requisitos que determinan el significado del hablante? Reconstruiremos la noción central del programa de Grice, la noción del significado del hablante, respondiendo a estas cuestiones.

Los términos en los que se definen, de un modo aproximado, las condiciones que posibilitan significar no naturalmente algo (esto es, el significado del hablante) son los siguientes:

(1) $\quad H$ debe tener la intención de producir una respuesta $r$ en $A$ (un auditorio) mediante la emisión de $x$.

(2) A debe reconocer la intención (1).

(3) Que (2) cuente como parte de las razones de A para realizar $r$.

Así, por ejemplo, para que la proferencia "hace un día estupendo" signifique "voy a ir a la playa" es necesario que el hablante que la profiere: (1) tenga la intención de hacer creer al oyente que va a ir a la playa, (2) que el oyente reconozca la intención del hablante de hacerle creer que va a ir a la playa, y (3) que el reconocimiento de la intención del hablante forme parte de las razones del oyente para creer que el hablante va a ir a la playa.

Esta definición recibió numerosas críticas y reformulaciones, entre las que destacan las de Strawson ${ }^{18}$, Searle ${ }^{19}$ y Schiffer ${ }^{20}$. Grice consideró y rebatió algunas de ellas de un modo razonablemente satisfactorio en su artículo de 1969. En cualquier caso, lo que nos interesa destacar de su caracterización es lo siguiente: afirmar que las condiciones necesarias para que $x$ signifique no naturalmente algo se determinan apelando a lo que los usuarios hacen cuando

\footnotetext{
${ }^{18}$ Strawson, P., "Intention and Convention in Speech Acts", en: The Philosophical Review, v. LXXIII, 4 (1964), pp. 439-460; traducción: "Intención y convención en los actos de habla", en: Strawson, P., Ensayos lógico-lingüísticos, Madrid: Tecnos, 1983.

${ }^{19}$ Searle, J., "What is a Speech Act?", en: Black, M. (ed.), Philosophy in America, Nueva York: Blackburn, 1965.

${ }^{20}$ Schiffer, S., Meaning, Oxford: Clarendon Press, 1972.
} 
profieren $x$, es explicar el significado del hablante en términos psicológicos. Estos son en cierto sentido independientes de las nociones semánticas, ya que no es necesario conocer el significado de una proferencia para reconocer las intenciones que definen el significado del hablante. Es de esta manera que Grice reduce la noción de significado no natural a las intenciones de los hablantes ${ }^{21}$. El significado ocasional del hablante se explica en términos de las intenciones del hablante y en términos del reconocimiento de las intenciones del hablante por parte del oyente.

El otro elemento relevante en su programa, como señalamos antes, es la racionalidad. En su trabajo "Lógica y conversación" de 1975, distinguió entre implicaturas convencionales e implicaturas conversacionales (no convencionales). En estas últimas lo implicado por un hablante al proferir $x$ ni es fruto de una convención lingüística, ni se sigue lógicamente del significado de $x$. Para explicar las implicaturas conversacionales es necesario apelar a una de las condiciones o principios que gobiernan nuestras conversaciones: la racionalidad. De acuerdo con Grice $^{22}$, nuestras prácticas comunicativas no son sucesiones de observaciones inconexas, sino una sucesión de proferencias conectadas entre sí, es decir, una sucesión de esfuerzos cooperativos que atienden a propósitos comunes marcados por los participantes de la conversación. Así, ser cooperativo es una forma de ser racional y es una pauta general que orienta los intercambios lingüisticos. Este principio que los hablantes siguen se denomina "Principio Cooperativo" (PC).

Este reza: "Haga usted su contribución a la conversación tal y como lo exige, en el estadio que tenga lugar, el propósito o la dirección del intercambio que usted sostenga"23. PC está constituido por cuatro categorías y nueve máximas. Las categorias son "Cantidad", "Cualidad", "Relación" y "Modo". La máxima más relevante de la categoria de cantidad es que se proporcione tanta información como sea requerida y no más. En la categoría de cualidad destaca la máxima de no decir aquello que se crea falso o (aquello) de lo que se carece de pruebas adecuadas. La categoría de relación se especifica con la máxima de que lo que se diga sea pertinente. Por último, la categoría de modo es relativa a cómo se dice algo, a diferencia de las anteriores que eran relativas a lo que

${ }^{21}$ Grice, H.P., "Las Intenciones y el Significado del Hablante", pp. 494-495.

${ }^{22}$ Grice, H.P., "Logic and Conversation", en: Cole, P. y J.L. Morgan (eds.), Syntax and Semantics, Volume 3: Speech Acts, Nueva York: Academic Press, 1975, pp. 41-58; traducción: "Lógica y conversación”, en: Valdés, L. (ed.), La búsqueda del significado, Madrid: Tecnos, 2005, p. 524.

${ }^{23}$ Ibid. 
se dice. En esta encontramos máximas como la de evitar ser oscuro o ambiguo al expresarse.

Estas máximas, nos dice Grice ${ }^{24}$, pueden dejar de cumplirse de varias maneras: (i) violando una de ellas, (ii) dejándola en suspenso, (iii) desembocando en una situación conflictiva, y (iv) dejando manifiestamente de cumplirla. Esta última situación es la que característicamente genera el fenómeno de las implicaturas conversacionales y en estos casos se está explotando una máxima. Teniendo en cuenta esto, ya podemos explicar la implicatura conversacional. El ejemplo utilizado es el siguiente: A y B están hablando de su amigo C que trabaja desde hace poco en un banco. A le pregunta a B cómo le van las cosas a C, y B profiere: (z) “iMuy bien! Se siente a gusto con sus compañeros y, además, no lo han metido todavia en la cárcel" 25 . Según Grice, para que un hablante H implique $q$ al decir $p$ satisfactoriamente (en el caso de una implicatura conversacional) es necesario que:

(1) $H$ observe el PC.

(2) $H$ piense que $q$.

(3) $H$ piense que el oyente es competente para captar que (2) es necesario.

Teniendo como base la cooperación, cuando $H$ dice $p$ ha dejado de ser cooperativo. Sin embargo, no seguiría siéndolo si hubiera querido decir $q$. $H$ sabe que el oyente es capaz de pensar que ha querido decir q. Luego, $H$ ha implicado $q$. En el ejemplo, la cosa sería como sigue: (1) B ha violado aparentemente la máxima de ser pertinente al proferir $z$, sin embargo, no hay razón para pensar que B ha dejado de ser cooperativo; (2) $z$ podria dejar de ser irrelevante si A considera que $\mathrm{B}$ piensa que $\mathrm{C}$ es potencialmente deshonesto; (3) B sabe que A es capaz de darse cuenta de (2). Por tanto, B está implicando que C es una persona potencialmente deshonesta.

Así es como el filósofo explica las implicaturas conversacionales, un fenómeno que pertenece al ámbito de lo que un hablante quiere decir con lo que dice. A modo de resumen: Grice, al caracterizar la noción fundamental de su propuesta, reduce los conceptos semánticos a nociones psicológicas, como las intenciones, y explica ciertos fenómenos de la comunicación -las implicaturas conversacionales- sobre la base de la racionalidad de los participantes (PC).

${ }^{24}$ Ibid., pp. 528-529.

${ }^{25}$ Ibid., p. 522. 


\section{Interpretando a Grice: el naturalismo de la intencionalidad}

Pueden distinguirse al menos dos estrategias opuestas en la tarea de esclarecer el funcionamiento de la comunicación. Una de estas estrategias -defendida por Austin, Searle, Wittgenstein, entre otros- sostiene que la comunicación puede explicarse en términos de prácticas institucionales convencionales, es decir, que las acciones comunicativas se explican apelando a prácticas gobernadas por reglas constitutivas. Por el contrario, la otra estrategia mantiene que la comunicación puede explicarse en términos de las actitudes proposicionales de los participantes, y que estas son independientes de cualquier práctica convencional. El proyecto de Grice puede interpretarse como un caso del segundo tipo de estrategia.

Como vimos más arriba, el significado puede explicarse en términos de las intenciones del hablante y esto quiere decir, aproximadamente, que las intenciones comunicativas de un hablante determinan el significado del hablante. Explicar las prácticas comunicativas en términos de las intenciones y creencias de los participantes -actitudes que pueden entenderse sin apelar a ningún tipo de convención- es reducir los conceptos semánticos a conceptos mentales intencionales y esto, de acuerdo con Stalnaker ${ }^{26}$, casa bien con la visión naturalista de la intencionalidad. Hay dos formas de ser naturalista con respecto a la intencionalidad:

(1) Naturalismo reduccionista: Defender que los fenómenos intencionales son explicables en términos de fenómenos estudiados desde otras ciencias, como la psicología. Este tipo de naturalismo suele denominarse "naturalismo epistemológico".

(2) Naturalismo compatibilista: Este sentido de naturalismo se compromete con que el tratamiento ofrecido de la intencionalidad no vaya en contra de lo que sostienen las ciencias, es decir, que haya continuidad con ellas. Este tipo de naturalismo suele denominarse "naturalismo metodológico".

Stalnaker afirma que el programa de Grice es compatible con una posición naturalista de la intencionalidad en sentido reduccionista ${ }^{27}$. Desde el punto de vista naturalista, el significado del hablante se explicaría apelando,

\footnotetext{
${ }^{26}$ Stalnaker, R., Context, Oxford: Oxford University Press, 2014, p. 40.

27 En Inquiry, Stalnaker trata el problema de la intencionalidad y defiende una posición naturalista en el sentido reduccionista.
} 
en palabras de Robert Thompson, a "una descripción psicológica de lo que literalmente ocurre en la cabeza de los hablantes cuando se comunican"28. Esta interpretación implica que las intenciones de un hablante (y, por tanto, lo que el hablante quiere decir) pueden explicarse en términos de la ciencia, esto es, en términos descriptivos. Esta interpretación descriptivista de la intencionalidad tiene partidarios desde antiguo ${ }^{29}$. Sin embargo, su modo de entender la intencionalidad también ha engendrado monstruos filosóficos dificiles de combatir.

Podemos situar el origen de esta interpretación tradicional de la intencionalidad en la obra de Brentano, Psychology from an Empirical Standpoint. Preocupado por hacer de la psicología una ciencia, Brentano distinguió entre fenómenos físicos y fenómenos mentales. En el que seguramente sea su pasaje más famoso, se define a estos últimos del siguiente modo: "Cada fenómeno mental está caracterizado por lo que los escolásticos de la Edad Media llamaron la inexistencia intencional (o mental) de un objeto, lo que puede llamarse, aunque no sin cierta ambigüedad, su referir a un contenido, su dirección hacia un objeto (que no debe ser entendido como una cosa) o su objetividad inmanente. Cada fenómeno mental contiene algo así como un objeto que no siempre se comporta del mismo modo. En la presentación hay algo que es presentado, en el juicio algo que es afirmado o negado, en el amor lo amado, en el odio lo odiado, en el deseo lo deseado y así sucesivamente"30.

Para Brentano, la inexistencia intencional, entendida como la cualidad de "referir a un contenido, su dirección hacia un objeto", es la marca de lo mental. Los estados mentales se caracterizan por apuntar a un objeto, su contenido, que posee inexistencia intencional, esto es, existe dentro del propio estado mental. En pocas palabras, los estados mentales están dirigidos hacia objetos que existen dentro de dichos estados mentales. Así, cuando un hablante $H$ está en un estado mental de creencia, de deseo, de temor, de expectativa o de intención, la relación de creencia, deseo, temor, expectativa o intención, conecta a $H$ con el contenido de su creencia, deseo, temor, expectativa o intención, siempre en el momento concreto del estado mental. Cuando creo que esta noche hará mucho frío, la relación de creencia me conecta, en el momento concreto en el que tengo dicha creencia, con el contenido de que esta noche hará mucho frío.

\footnotetext{
${ }^{28}$ Thompson, J., "Still Relevant: HP Grice's Legacy in Psycholinguistics and Philosophy of Language", en: Teorema: International Journal of Philosophy, v. XXVI, 2 (2007), p. 102.

${ }^{29}$ Moran, D., "Intentionality: Some Lessons from the History of the Problem from Brentano to the Present", en: International Journal of Philosophical Studies, v. XXI, 3 (2013), pp. 317-358.

${ }^{30}$ Brentano, F., Psychology from an Empirical Standpoint, McAlister, L. y P. Kegan (eds.), Londres: Routledge, 1995, p. 88.
} 
Independientemente de que esta noche haga mucho frío o no, que esta noche hará mucho frio es el objeto intencional con el que estoy en la relación de creencia. En términos de la teoría de Grice: cuando $H$ tiene la intención de que $p$ (la intención de que su oyente crea algo), la relación de intención conecta a $H$ con el contenido $p$. Ahora bien, ¿qué son estos contenidos intencionales? Este es uno de los monstruos filosóficos de los que advertíamos antes.

Al margen de la naturaleza de los contenidos mentales, la interpretación naturalista de las intenciones se encuentra con otro problema: la comúnmente denominada "falacia descriptivista". De acuerdo con Macarthur ${ }^{31}$, la falacia descriptivista consiste en pensar que todas las oraciones en indicativo de nuestro lenguaje cumplen siempre una función descriptiva. Sin embargo, en la comunicación utilizamos cierto tipo de oraciones cuyo significado no describe nada del mundo y que, por tanto, no pueden reducirse a un conjunto de descripciones. Una de las posiciones filosóficas que pone especial énfasis en esta idea es el expresivismo. En la siguiente sección abordaremos esta teoría y expondremos sus principales argumentos contra el naturalismo reduccionista.

\section{Los argumentos del expresivismo clásico contra el naturalismo}

El expresivismo es una postura semántica relativa al tratamiento de los términos y enunciados morales que comenzó a desarrollarse en el siglo XX. A pesar de que en la actualidad bajo la etiqueta "expresivismo" caen propuestas muy dispares entre sí, y que su análisis se ha extendido a otros ámbitos del discurso más allá del concerniente a la ética, en todos estos diagnósticos encontramos una tesis compartida: la idea de que describir o representar estados de cosas no es la única función que cumple el lenguaje. Esta tesis negativa definitoria del expresivismo permite afirmar que se trata de una teoría de corte pragmatista anti-representacionalista. La etiqueta "pragmatista" se debe a que defender, como hace el expresivismo, que el lenguaje no se utiliza exclusivamente para describir o representar fragmentos de la realidad encaja a la perfección con las observaciones paradigmáticas que iniciaron el desarrollo de la corriente pragmática en filosofia del lenguaje. Las siguientes palabras famosas de J. L. Austin sirven como ejemplo: "Durante mucho tiempo los filósofos han presupuesto que el papel de un 'enunciado' solo puede ser 'describir' algún estado de cosas, o 'enunciar algún hecho', con verdad o falsedad....

${ }^{31}$ Macarthur, D., "Wittgenstein and Expressivism", en: Whiting, D. (ed.), The Later Wittgenstein on Language, Basingstoke: Palgrave Macmillan, 2010, p. 81. 
las 'proposiciones éticas' quizá persiguen manifestar emociones, exclusiva o parcialmente, o bien prescribir conducta o influirla de maneras especiales" ${ }^{\prime 2}$.

Por otra parte, abrir la puerta a un tratamiento no descriptivo para determinados enunciados supone atacar de un modo muy directo las intuiciones del representacionalismo. La idea básica de una teoría representacionalista es que cualquier entidad susceptible de propiedades semánticas es significativa en virtud del estado de cosas que representa. En otras palabras, las creencias, los términos o las oraciones representan, refieren, corresponden, o se hallan causalmente conectadas con objetos o propiedades de objetos ${ }^{33}$. En este sentido, el expresivismo es una postura anti-representacionalista, pues defiende que hay ciertos enunciados significativos que no describen o representan partes del mundo. Cabe aclarar que, aun cuando reconoce una función no descriptiva para ciertas expresiones, el expresivista no rechaza el representacionalismo en su totalidad necesariamente. Una postura expresivista puede ser compatible con un tratamiento descriptivista de otros enunciados. El caso del primer Wittgenstein sirve como ejemplo de esta compatibilidad: en el Tractatus, Wittgenstein propuso un análisis representacionalista de las oraciones atómicas y un diagnóstico expresivista para las constantes lógicas. Por tanto, la tesis común (TC) a todas las posturas expresivistas puede formularse del siguiente modo:

[TC] Ciertas expresiones significativas no describen ni representan estados de cosas.

Ahora bien, ¿qué expresan las expresiones que no hablan de cómo es el mundo? ¿Cuáles son sus contenidos? ¿Son de tipo fáctico o normativo? Los distintos tipos de expresivismo que han ido desarrollándose en los últimos dos siglos se diferencian unos de otros a partir del tipo de respuesta que ofrecen para estas cuestiones. De un modo general, desde una postura expresivista ética clásica puede decirse que estas expresiones expresan los sentimientos o la aprobación de los hablantes. Cuando un hablante profiere "tengo miedo" o "robar es malo" (casos paradigmáticos del expresivismo), un expresivista clásico diría que, en el primer caso, el hablante está expresando mediante dicha expresión sus emociones y, en el segundo, el hablante está mostrando su desacuerdo con

\footnotetext{
${ }^{32}$ Austin, J., Cómo hacer cosas con palabras, Carrió, G.R. y E.A. Rabossi (trads.), Barcelona: Paidós, 1967, p. 21.

${ }^{33}$ Frápolli, M.J. y N. Villanueva, "Frege, Sellars, Brandom: expresivismo e inferencialismo semánticos”, en: Pérez Chico, D. (ed.), Perspectivas en la filosofia del lenguaje, Zaragoza: Prensas Universitarias de Zaragoza, 2013, p. 583.
} 
respecto a robar ${ }^{34}$. Así, cómo se debe entender el contenido de las expresiones no descriptivas es lo que ha dado lugar a las distintas variantes contemporáneas de expresivismo. Como ejemplos de posturas expresivistas contemporáneas sirven el cuasi-realismo de Blackburn, el expresivismo lógico de Sellars y Brandom, el expresivismo ecuménico de Ridge, el expresivismo realista de Copp, el expresivismo normativo de Gibbard, el neo-expresivismo de Bar-On y Finkelstein, el expresivismo global de Price, el expresivismo epistemológico de Chrisman, el expresivismo comunitario de Glock, el expresivismo mínimo de Frápolli y Villanueva, etcétera.

El expresivismo clásico como teoría semántica está relacionado en su inicio con el análisis de los términos y enunciados éticos. Se trata, por tanto, de una posición meta-ética que intenta responder las siguientes cuestiones: ¿cuál es el significado de términos como "bueno", "malo", "correcto", entre otros, y qué significan expresiones como "es bueno ayudar a los demás", "robar es malo" o "hay que comportarse correctamente"? El punto de partida de esta posición es la observación de que este tipo de términos y enunciados, que son morales, no se comportan del mismo modo que otros términos y oraciones sintácticamente idénticos. Las palabras "azul", "roto" y "puntiagudo", y las expresiones "el bote es azul", "el plato está roto" o "la aguja es puntiaguda" pueden explicarse apelando a representaciones de estados de cosas. Estas expresiones describen fragmentos de la realidad o hechos, y su verdad o falsedad depende de su correspondencia con el mundo. Sin embargo, las expresiones del tipo "es bueno ayudar a los demás" o "robar es malo" no parece que describan ningún estado de cosas. ¿Qué hecho corresponde, y por tanto hace verdadero o falso, a estas afirmaciones? De esta disanalogía del lenguaje entre enunciados descriptivos y no descriptivos emergen las posiciones expresivistas, haciendo énfasis en la denominada "falacia descriptivista", que consiste en pensar que la única función del lenguaje es describir estados de cosas.

El origen de esta posición meta-ética, como señalan Frápolli y Villanueva ${ }^{35}$, se remonta hasta las consideraciones de Hume en su obra Tratado sobre la naturaleza humana (1739-1740). Hume ya señaló, con la famosa falacia naturalista, que las oraciones normativas no pueden reducirse a un conjunto de descripciones: del ser no se sigue el deber ser. Moore, por su parte, sostuvo algo parecido: los predicados normativos son primitivos $\mathrm{y}$, por tanto, no pueden

\footnotetext{
${ }^{34}$ En la literatura reciente se diria que la proferencia emitida por un hablante expresa el estado mental o los compromisos del hablante.

${ }^{35}$ Ibid.
} 
definirse apelando a predicados más básicos. Entre los diagnósticos de las obras de Ogden y Richards (1923), Barnes (1933), Ayer (1936) y Stevenson (1937), y las posturas prescriptivistas de Carnap (1937) y Hare (1955), hay un lugar común en esta línea: si bien no todos estos autores pueden clasificarse como expresivistas, ellos consideran que el ámbito del discurso moral no tiene una función descriptiva y que sus enunciados no tienen valor de verdad.

Según Ayer, las oraciones relativas a la ética no tienen significado factual (no describen nada del mundo ni tienen condiciones de verdad), al contrario de lo que ocurre en las afirmaciones típicas de la ciencia. Por tanto, enunciados del tipo "robar es malo" expresan los sentimientos del hablante; en este caso, sus sentimientos de desaprobación. Stevenson ofreció un análisis similar, aunque más elaborado: distinguió dos usos distintos del lenguaje, el uso descriptivo y el uso dinámico, donde el último de estos tiene como objetivo expresar actitudes o sentimientos y mover a la acción. Para estos autores, representantes del expresivismo ético clásico, los términos y los enunciados relativos a la moral no están conectados ni con partes de estados de cosas, los primeros, ni con estados de cosas completos, los segundos: "no refieren a objetos ni a propiedades o relaciones identificables en los hechos reales o posibles. Por ello... las oraciones de la ética no se pueden usar para hacer aseveraciones verdaderas o falsas" ${ }^{36}$. Así, la tesis positiva que el expresivismo ético clásico añade a TC es la siguiente:

[TE]: Algunos términos y enunciados (los morales) no cumplen una función descriptiva, sino que más bien expresan los sentimientos o compromisos del hablante.

La unión de TC y TE caracteriza al expresivismo ético clásico. Aquellos términos y enunciados que pertenecen al ámbito de la moral son no descriptivos, y se caracterizan por expresar los sentimientos y sensaciones del hablante o por incitar a la acción. Esta propuesta permite explicar, entre otras cosas, por qué podemos seguir preguntando si algo es bueno a pesar de que se nos ofrezcan infinitas descripciones -el denominado "Argumento de la pregunta abierta"-, y por qué hay desacuerdo moral persistente. Cuando profiero "la filosofia es buena", puedo tener la intención de mostrar mi aprobación por la filosofía o incitar a alguien a que se inmiscuya en la lectura y reflexión filosófica, pero en ningún caso estoy describiendo nada, ni estados de cosas, ni partes del mundo, ni propiedades, ni nada similar; simplemente estoy evaluando, expresando, o animando a hacer tal o cual cosa a alguien. Debido a esto, alguien podría,

36 Ibid., p. 591. 
adoptando una actitud bastante incómoda, insistir preguntando por qué la filosofia es buena, o podría estar en desacuerdo conmigo. Cuando digo "la filosofia es buena", de acuerdo con la creencia generalizada en el expresivismo ético clásico, no estoy diciendo nada verdadero o falso. $\mathrm{Y}$ es en este sentido en el que se ha considerado al expresivismo clásico como no-factualista y nocognitivista. Ser no-factualista significa comprometerse con que los términos o enunciados relativos a la ética no tienen valor de verdad ${ }^{37}$.

Al margen del ámbito de la ética también han surgido posturas que encajan dentro del expresivismo clásico. El análisis anti-descriptivista fuera del entorno de la moral más destacable es quizás el que ofreció Wittgenstein, tanto en sus escritos tempranos como en su etapa más madura. En el Tractatus, sostuvo un tratamiento expresivista de las nociones de creencia, negación, y de las demás operaciones lógicas, pues mantuvo que estas expresiones (y las inferencias que realizamos con ellas) no representan hechos del mundo ni estados de cosas. En las Investigaciones filosóficas también encontramos un diagnóstico no descriptivista, esta vez acerca del significado de ciertas adscripciones de estados mentales (como las creencias, los deseos, las expectativas, etcétera). Para Wittgenstein, el contenido de expresiones del tipo "creo que mañana lloverá", "espero que él llegue" o "deseo comer una manzana", no son representaciones de hechos o partes del mundo. El hecho de que mañana llueva, que él llegue o que me coma una manzana, no hace verdadera mi creencia, ni cumple mi expectativa, ni satisface mi deseo, respectivamente. El contenido de este tipo de estados mentales es lingüístico. La expresión "creo que mañana lloverá" es equivalente a "tengo la creencia que se hace verdadera por el hecho de que mañana llueva". En otras palabras, la expresión "creo que $p$ " es equivalente a la expresión "tengo la creencia que se hace verdadera por el hecho de que $p$ ”, y no hay nada más que decir: "En el lenguaje se tocan expectativa y cumplimiento"38. Además de este análisis anti-representacionalista de la intencionalidad, Wittgenstein utilizó otro tipo de argumentos contra el carácter descriptivo de estos estados mentales como, por ejemplo, el argumento de la duración. Según este argumento, el hecho de que la duración de un estado mental sea irrelevante en cuanto a su significado indica que la expresión de estos no describe nada del mundo, sino

\footnotetext{
${ }^{37}$ La discusión acerca de qué significa que una expresión tenga valores de verdad y acerca de cuáles son exactamente los compromisos de una posición no-factualista excede completamente nuestros propósitos para este trabajo. No obstante, es probable que para defender que las expresiones no-descriptivas tienen valores de verdad simplemente sea necesario especificar que no se tiene una teoria de la verdad robusta.

${ }^{38}$ Wittgenstein, L., Investigaciones Filosóficas, § 445.
} 
que muestran nuestros compromisos, aquello que estamos dispuestos a hacer o decir. En Zettel, defendió explícitamente esta idea: si digo "espero que vengas" y alguien pregunta cuánto tiempo llevo esperando, en caso de que pudiese dar una respuesta razonable, dicha respuesta sería totalmente irrelevante para el fin (y por tanto para el significado) de mi expresión ${ }^{39}$.

Uno de los argumentos más potentes del expresivismo clásico, como ya hemos señalado, se lo debemos a Moore, el comúnmente denominado "Argumento de la pregunta abierta" (PA). Según PA, para cualquier definición $x$ que ofrezcamos de un término moral del tipo "bueno", alguien podría seguir preguntando “¿pero es $x$ bueno?”, sin que podamos satisfacer completamente la pregunta. La idea de PA es que sin importar la cantidad de descripciones que utilicemos para caracterizar una situación, la pregunta “¿pero es $x$ bueno?" seguirá siendo pertinente. Este argumento muestra que los términos normativos o morales no pueden reducirse a términos naturales, y esto se debe a que, cuando usamos los primeros no describimos partes del mundo, sino que las evaluamos. Otro de los argumentos más relevantes del expresivismo es el "Argumento del desacuerdo moral" (DM). Según DM, el desacuerdo moral es una evidencia de que los juicios morales no son juicios descriptivos. Que habitualmente discutamos sobre si tal cosa es buena o no sin lograr llegar a un lugar común muestra que tales consideraciones no son acerca de cómo son las cosas en el mundo, sino acerca de los compromisos de los hablantes u otras cosas, según el tipo de desacuerdo ${ }^{40}$.

Sin embargo, el argumento contra el carácter descriptivo de las atribuciones de estados mentales que nos parece más potente es el "Argumento de la duración” (D), ofrecido por Wittgenstein. De acuerdo con D, los estados mentales del tipo "espero que vengas" no tienen una duración genuina, es decir, es irrelevante con respecto al significado de la adscripción del estado mental preguntar algo como “¿cuánto tiempo llevas esperando?”. Por tanto, estas adscripciones de estados mentales no describen cómo es el mundo -pues no tienen duración-, sino que las usamos para manifestar algo ${ }^{41}$.

Todos estos argumentos (PA, DM y D) vienen a mostrar las debilidades del naturalismo de la intencionalidad en sentido reduccionista. Si se interpreta en

\footnotetext{
39 Wittgenstein, L., Zettel, § 78.

40 El tema del desacuerdo es uno de los temas filosóficos más trabajados actualmente y se han ofrecido numerosas propuestas acerca de cómo explicar qué ocurre cuando dos sujetos se encuentran en un desacuerdo evaluativo. El reciente libro de John MacFarlane, Assessment Sensitivity (Oxford: Clarendon Press, 2014), es una buena prueba de ello.

${ }^{41}$ Wittgenstein, L., Zettel, § 78.
} 
estos términos la teoria de Grice, resulta dificil defender el beneficio de reducir la semántica a nociones psicológicas. Si debemos explicar el significado de lo que los usuarios quieren decir apelando en última instancia a sus intenciones (y, por tanto, a sus estados mentales), y entendemos esto como un tipo de naturalismo en sentido reduccionista, entonces no conseguimos explicar el significado de algunas cosas que proferimos cuando nos comunicamos. El significado de las intenciones que no pueden reducirse a un conjunto de descripciones quedaria fuera.

\section{Interpretando a Grice: otra posibilidad}

De acuerdo con Stalnaker ${ }^{42}$, cabe otro modo de interpretar la teoría de Grice. En esta sección defenderemos que, para interpretar correctamente el programa de Grice, no hay que obviar uno de sus elementos fundamentales, a saber, PC. Señalando el papel central que juega este principio en la comunicación, ofreceremos otra interpretación de Grice que se aleja de la interpretación naturalista. Esta última, como ya vimos, reduce el significado del hablante (el significado no natural) a las intenciones comunicativas, e interpreta estas en términos de la ciencia. Sin embargo, para que el esquema griceano funcione, argumentaremos, es necesario tener presente PC. Consideremos la siguiente caracterización que Grice propuso en 1957 sobre el significado no natural: "A significa no naturalmente algo con $x$ " es aproximadamente equivalente a "A profirió $x$ con la intención de inducir una creencia mediante el reconocimiento de su intención"43.

Ahora bien, cuando un agente $A$ profiere $x$ significando no naturalmente $p$, de acuerdo con Stalnaker ${ }^{44}$, podemos hacer dos preguntas sobre este acto:

a) ¿Por qué proferir $x$ es un modo de que el oyente llegue a reconocer la intención del hablante de inducirle la creencia $p$ ?

b) ¿Por qué el hecho de que el oyente llegue a reconocer la intención del hablante de inducirle la creencia $p$ es una forma de hacerle creer $p$ ?

Una respuesta razonable para (a) sería apelar al lenguaje entendido en términos de prácticas convencionales. Si concebimos el lenguaje en sentido normativo, podemos decir que proferir algo es una manera de decir lo que uno

42 Stalnaker, R., Context, p. 39.

43 Grice, H.P., "Significado", p. 484.

44 Stalnaker, R., Context, p. 40. 
quiere decir. Pero las condiciones constitutivas del significado no natural son independientes de la respuesta que demos a esta pregunta. Que proferir $x$ sea una manera en la que un hablante diga lo que quiere decir no responde a cuáles son las condiciones que permiten a un hablante significar no naturalmente algo. La pregunta (b) apunta en esta dirección. Para que el reconocimiento por parte del oyente de la intención del hablante de hacerle creer $p$ sea una manera de que el oyente crea $p$ debe haber un "patrón de interés común" 45 . El reconocimiento de la intención del hablante será una razón para que el oyente crea $p$ solamente si el oyente cree que el hablante quiere hacerle creer la verdad. Por tanto, el éxito del diagnóstico de Grice depende de esta expectación mutua entre los participantes, y es precisamente aquí donde entra en juego PC.

Para Grice, nuestras prácticas comunicativas no son sucesiones de observaciones inconexas, sino sucesiones de proferencias conectadas entre sí, esto es, continuos esfuerzos cooperativos marcados por los propósitos comunes de los participantes. Esto es precisamente a lo que apunta PC con la prescripción "Haga usted su contribución a la conversación tal y como lo exige, en el estadio que tenga lugar, el propósito o la dirección del intercambio que usted sostenga" y sus máximas. Teniendo en cuenta todo esto, y de acuerdo con Stalnaker ${ }^{46}, \mathrm{PC}$ es un requisito necesario en torno al que se articula la definición griceana que hemos considerado sobre el significado no natural (significado del hablante). Y que tal principio normativo esté en la base -y sea una condición necesaria- del programa de Grice es un argumento razonablemente potente contra la interpretación naturalista de su diagnóstico. Desde este punto de vista, pueden rastrearse pasajes en sus escritos donde él mismo toma distanciamiento del naturalismo psicológico. El siguiente es un buen ejemplo: "Niego tener la intención de poblar toda nuestra vida verbal con ejércitos de complicados sucesos psicológicos. No pretendo resolver ningún enigma filosófico sobre las intenciones, pero quiero argüir brevemente que no surge ninguna dificultad especial a partir de mi uso de la palabra 'intención' en relación con el significado" 47 .

No tener la intención de introducir "complicados sucesos psicológicos" como partes de su proyecto, no pretender "resolver ningún enigma filosófico sobre las intenciones" y no reconocer dificultad con el "uso de la palabra 'intención' en relación con el significado", a nuestro modo de ver, son declaraciones explícitas contra la empresa de explicar las intenciones a la

45 Ibid., p. 41.

${ }^{46}$ Ibid., p. 42.

${ }^{47}$ Grice, H.P., "Significado", p. 489.

ARETÉ Revista de Filosofia, vol. XXIX, № 1, 2017 / ISSN 1016-913X 
manera naturalista. Sería razonable que alguien dudase de la legitimidad de interpretar así estas palabras, debido a que pertenecen al primer artículo donde Grice expuso su análisis y PC no apareció en consideración hasta 1975. Alguien podría pensar que lo que ocurre es que el filósofo cambió de parecer a lo largo de su vida académica. Sin embargo, en el "Retrospective Epilogue" (1987) de su obra Studies in the Way of Words (1989), Grice señala que: "Las conexiones entre los temas discutidos [en sus artículos] son a veces más fuertes e interesantes de lo que los propios artículos dejan claro: en parte debido a que, supongo, no vi estas conexiones en el momento en el que los ensayos fueron escritos, y únicamente al mirar atrás empiezo a ver la cantidad [de conexiones] y su importancia"48.

Ver con mayor claridad la cercanía y la importancia de los distintos temas tratados en retrospectiva no significa no haberlas reconocido en un principio (o al menos no sospechar algo). En cualquier caso, reconocer la conexión, antes o después, entre los temas tratados es una forma de fortalecer nuestra interpretación. Por si quedan dudas, hay un fragmento todavía más claro donde Grice reconoce la importancia de PC para explicar la comunicación, lo que, en consecuencia, lo aleja explícitamente de la reducción naturalista: "Esta tesis [el uso del lenguaje es una actividad racional] toma forma más concretamente defendiendo que el tipo de actividad racional que envuelve el uso del lenguaje es una forma de cooperación racional"49.

Así, tanto la necesidad de un interés común (PC) para que el reconocimiento de la intención de un hablante de hacer creer $p$ a un oyente cuente como razón para que el oyente crea $p$, como los pasajes citados de Grice, apoyan nuestra interpretación no reduccionista del programa de Grice. Bajo esta interpretación pueden eludirse todas las objeciones contra la interpretación naturalista consideradas en este trabajo.

\section{Conclusión}

Como hemos visto, el programa de Grice supone una reducción de las nociones semánticas a nociones intencionales. Esta reducción casa bien con el naturalismo de la intencionalidad. Sin embargo, interpretado así, el programa griceano genera más problemas que beneficios. Hay otra interpretación

\footnotetext{
${ }^{48}$ Grice, H.P., "Meaning Revisited", en: Grice, H.P., Studies in the Way of Words, Cambridge: Harvard University Press, 1989, p. 339.

49 Ibid., p. 341.
} 
posible, incompatible con la interpretación naturalista de la intencionalidad: la interpretación que señala la necesidad de PC para la explicación griceana de la comunicación. Dado que PC es un principio normativo y que la explicación del significado del hablante (significado no natural) requiere de PC, esta interpretación es incompatible con el naturalismo reduccionista. Esta segunda interpretación esquiva las críticas del expresivismo y, además, pone sobre la mesa el error que supone interpretar a Grice a la luz del naturalismo.

\section{Bibliografia}

Ayer, A.J., Lenguaje, Verdad y Lógica, Barcelona: Ediciones Orbis, 1936/1984.

Bach, K. y R. Harnish, Linguistic Communication and Speech Acts, Cambridge: MIT Press, 1982.

Brentano, F., Psychology from an Empirical Standpoint, 1874; edición en inglés McAlister, L. y Kegan, P. (eds.), Londres: Routledge, 1973; reimpresa con una introducción de Simons, P., London: Routledge, 1995.

Crane, T., Objects of Thought, Oxford: Oxford University Press, 2013. https://doi. org/10.1093/acprof:oso/9780199682744.001.0001

Frápolli, M. J. y E. Romero, Una aproximación a la filosofia del lenguaje, Madrid: Sintesis, 1998.

Frápolli, M. J. y N. Villanueva, "Frege, Sellars, Brandom: expresivismo e inferencialismo semánticos”, en: Pérez Chico, D., Perspectivas en la filosofia del lenguaje, Zaragoza: Prensas Universitarias de Zaragoza, 2013, pp. 583-617.

Fodor, J., A Theory of Content and Other Essays, Cambridge: MIT Press, 1990.

Grice, H.P., "Meaning", en: The Philosophical Review, v. LXVI (1957), pp. 377-388, Traducción: "Significado", en Valdés, L. (ed.), La búsqueda del significado, Madrid: Tecnos, 2005.

Grice, H.P., "Utterer's Meaning and Intentions", en: The Philosophical Review, v. LXXVIII (1969), pp. 147-177. Traducción: "Las intenciones y el significado del hablante", en: Valdés, L. (ed.), La búsqueda del significado, Madrid: Tecnos, 2005.

Grice, H.P., "Logic and Conversation", en: Cole, P. y J.L. Morgan (eds.), Syntax and Semantics. Speech Acts, v. III. Academic Press, 1975. Traducción: "Lógica y Conversación”, en: Valdés, L. (ed.), La búsqueda del significado, Madrid: Tecnos, 2005

Grice, H.P., "Meaning Revisited", en: H. P. Grice, Studies in the Way of Words, Cambridge: Harvard University Press, 1989.

Grice, H.P., "Retrospective Epilogue", en: H. P. Grice, Studies in the Way of Words, Cambridge: Harvard University Press, 1989.

Kölbel, M., "Conversational Score, Assertion, and Testimony", en: Brown, J. y H. Cappelen (eds.), Assertion: New Philosophical Essays, Oxford: Oxford University Press, 2011, pp. 49-77. https://doi.org/10.1093/acprof:oso/9780199573004.003.0003

Lewis, D., Convention, Cambridge: Harvard University Press, 1969. 
Macarthur, D., "Wittgenstein and Expressivism", en Whiting, D. (ed.), The Later Wittgenstein on Language, Basingstoke: Palgrave Macmillan, 2010.

Moore, G.E., Principia Ethica, Prometheus Books, 1903/1988.

Moran, D., "Intentionality: Some Lessons from the History of the Problem from Brentano to the Present", en: International Journal of Philosophical Studies, v. XXI, 3 (2013), pp. 317-358. https://doi.org/10.1080/09672559.2013.812605

Schiffer, S., Meaning, Oxford: Clarendon Press. 1972.

Searle, J., "What is a Spech Act?", en: Black, M. (ed.), Philosophy in America, Nueva York: Blackburn, 1965, Traducción en: Valdés, L. (ed.), La búsqueda del significado, Madrid: Tecnos, 2005.

Sperber, D. y D. Wilson, Relevance: Communication and Cognition, Oxford: Blackwell, 1986.

Stalnaker, R., Inquiry, Cambridge: MIT Press, 1984.

Stalnaker, R., Context, Oxford: Oxford University Press, 2014. https:/ /doi.org/ 10.1093/ acprof:oso/9780199645169.001.0001

Stevenson, C.L., "The Emotive Meaning of Ethical Terms" en: Mind, New Series, v. XLVI, 181 (1937), pp. 14-31.

Stevenson, C. L., Ética y Lenguaje, Buenos Aires: Paidós, 1944.

Strawson, P., "Intention and Convention in Speech Acts" en: The Philosophical Review, v. LXXIII, 4 (1964), pp. 439-460. Reimp. en Strawson, P., Logico-Linguistic Papers. Methuen. Londres. Traducción: "Intención y Convención en los Actos de Habla", en: Ensayos lógico-lingüísticos, Madrid: Tecnos, 1983.

Thompson, J., "Still Relevant: HP Grice's Legacy in Psycholinguistics and Philosophy of Language", en: Teorema: International Journal of Philosophy, v. 26 (2) (2007), pp. 77-109.

Wittgenstein, L., Tractatus logico-philosophicus, Madrid: Alianza Editorial, 1922/1957. Wittgenstein, L., Investigaciones Filosóficas, México: Universidad Nacional Autónoma de México, 1953/1986.

Wittgenstein, L., Zettel, México: Universidad Nacional Autónoma.

Recibido: 04/04/2016

Aceptado: 27/11/2016 